\title{
Psychotherapy at a Time of Diminishing Resources
}

\author{
I. F. Macilwain, Consultant Psychiatrist, Netherne Hospital, Coulsdon, Surrey
}

Estimating demand for psychotherapy is like trying to evaluate the size of a mountain range from the foothills- the higher one climbs, the more potential needs become apparent. The unspoken dream that at some point all these needs will be met by trained psychotherapists seems firmly destined to stay in the realms of fantasy. In reality, specialist psychotherapists will always be thin on the ground. In Surrey, where my own practice is based, I am, and am likely to remain, the only medically trained psychotherapist with four sessions allocated to a population of 200,000 . The psychotherapy department at St. George's Hospital, Tooting, to which I am also attached, has a catchment which extends to the whole South-West Thames Region, encompassing places as far afield as Chichester and Haywards Heath.

How can we best deploy these scanty resources and where should we draw the boundary with the private sector? These are difficult questions to answer, posing as they do a number of ethical and theoretical problems. I cannot claim to have any answers, but only, hopefully, to stimulate further discussion.

At present, demand is largely regulated by adopting a low profile-focusing on staff training rather than direct treatment and accumulating lengthy waiting lists for assessment and treatment. This is not to my mind a very satisfactory situation. No attempt is made to assess relative need, and those seeking personal growth experiences tend to receive as much help as the severely disturbed or personality disordered. At the same time, even within the same district, there are huge discrepancies in referral rates between different general practices. The fear of being overwhelmed by potential demand tends to prevent this from being tackled at source, and as a result, within my own district, address is the factor which probably correlates most highly with the likelihood of receiving specialist psychotherapeutic help.

To what extent can we look to our colleagues in other disciplines to meet the shortfall? Psychologists vary greatly in their training and experience. Although they appear to be moving away from a rigid behaviourist position, there are still relatively few with full psychodynamic or analytic training. Social workers are often very well trained, but expected to wear too many hats to be able to make much time available for time-intensive treatment of this sort. Nursing staff, although frequently interested and willing to train, rarely receive the support or opportunity they need to use their newly acquired skills. Art therapists, often among the best trained of the paramedical disciplines, often lack the professional credibility to use the skills they possess. Neither can we look to general psychiatrists to meet the demand. Consultants are frequently overloaded with administrative responsibilities and even if interested, lack the training to do more than brief supportive work. Junior staff are increasingly committed to rotation schemes which make long-term contracts unrealistic, with the exception of designated training cases required by the College. College policy on non-training grades is slowly erod- ing their numbers and making them less available than formerly to do work, the essence of which is continuity.

One is forced to conclude that there will never be sufficient personnel to address the task. If we are not to abandon the dream of providing a service for all who require it, it is clear that answers must be sought either at the selection stage or by modifying the treatment approach.

If we are to become more selective, who should we eliminate? Perhaps those with relationship difficulties and without a 'psychiatric' presentation should be referred to the private sector or refused treatment. Alternatively, they should not be given the option of individual therapy, but offered group therapy or nothing.

Regarding the treatment approach, there is a great need for experimentation with different formats, frequencies and models of treatment. I make a plea to all those who believe they have pioneered an approach in this area of treatment to publish it, no matter how briefly. Outcome data is badly needed to supplement the paucity of information currently available.

In East Surrey we have experimented with a time-limited daily group held in a day hospital. This involved each patient in a three-month commitment, with the leaving date set at the outset, and the processes of attachment and separation a major focus of the work. Five staff of different disciplines, including myself, acted as therapists, two being present in the group on any day, one of whom had also been present the previous day, to ensure continuity. Results were encouraging. A number of patients who had proved intransigent to other approaches showed a significant shift. A detailed outcome study will be completed shortly. I also have a number of quite disturbed patients who have responded well to a regime of one hour long interview per month. In this way regressive dependence is avoided, insights are taken away and incorporated into daily life, and continuity can be maintained over a long period with minimal use of time.

To me, group approaches offer the best chance of compensating for the inadequacies of the system. They offer opportunities for training therapists which are unparalleled, and provided the approach is group centred rather than therapist centred, the patients will act as culture carriers, allowing the group to tolerate periodic changes of one of the therapists. Also, in my view, the inexperienced therapist can do far less damage in a group, provided he is paired with a more experienced person, of whatever discipline.

Finally, the specialist psychotherapist can encourage and support the psychotherapeutic activities of a whole range of disciplines referred to above, taking a responsibility for quality control and staff training where necessary. This will hopefully free some of his own time for exploring and developing contacts with the primary care team, who, it should never be forgotten, do more counselling than all the specialists put together! 